\title{
CERTAIN HILBERT SPACES OF ENTIRE FUNCTIONS
}

\author{
BY D. J. NEWMAN AND H. S. SHAPIRO \\ Communicated by L. Cesari, March 31, 1966
}

1. Introduction. The research reported on in the present note was motivated by the following Proposition (F), due to Ernest Fischer ([5], see also [4] for an earlier version; actually Fischer proved a more general result, but the special case suffices as a point of departure for our discussion):

(F) Let $P$ denote a homogeneous polynomial in $z_{1}, \cdots, z_{k}$ with complex coefficients. Then every polynomial in $z_{1}, \cdots, z_{k}$ has a unique representation $Q P+R$ where

$$
P^{*}\left(\frac{\partial}{\partial z_{k}}, \ldots, \frac{\partial}{\partial z_{k}}\right) R=0
$$

Here $Q, R$ denote polynomials in $z_{1}, \cdots, z_{k}$ and $P^{*}$ denotes the polynomial whose coefficients are the complex conjugates of those of $P$.

The proposition (F) underlies various formal schemes for exhibiting a basic set of polynomial solutions of a partial differential equation. (Compare Horváth [8], and for the special case $P=z_{1}^{2}+z_{2}^{2}+z_{3}^{2}$ Hobson [7, Chapter IV]).

Now, if the word "homogeneous" is suppressed, (F) becomes false, since indeed in that Case (1) need not have any nonnull polynomial solution. Nevertheless, if we are willing to abandon the realm of polynomials ( $\mathrm{F}$ ) can be extended. The clue as to how to proceed is provided by Fischer's proof of $(\mathrm{F})$ : he defines in the linear manifold of polynomials an inner product with respect to which the operator "multiplication by $P^{\prime}$ and the differential operator $P^{*}\left(\partial / \partial z_{1}, \cdots\right.$, $\left.\partial / \partial z_{k}\right)$ are adjoint to one another; the required decomposition is then just the orthogonal complement decomposition of the space induced by a pair of adjoint operators. Of course, the polynomials do not form a (complete) Hilbert space with respect to Fischer's inner product. Their completion turns out to be a certain space $F_{k}$ of entire functions of order two. Within $F_{k},(\mathrm{~F})$ is now true also in the case that $P$ is not homogeneous, and indeed even for certain entire transcendental functions $P$, with a suitable interpretation of $P^{*}\left(\partial / \partial z_{1}, \cdots\right.$, $\left.\partial / \partial z_{k}\right)$. The extension of Fischer's result to $F_{k}$ is far more difficult, however, than the proof of $(\mathrm{F})$ insofar as the problem in $F_{k}$ is closely intertwined with a series of questions which have no counterpart in the polynomial case. These questions concern adjoints of unbounded 
operators, weighted polynomial approximation of holomorphic functions, and infinite-order differential equations.

We proceed now to the statement of our main results. Proofs of these results have been published in the form of mimeographed notes [13], and a revised version of this material is currently being prepared for publication. The physicist V. Bargmann has, independently of the preceding considerations, been led to study the spaces $F_{k}$ (see [2]). There is little overlap between Bargmann's work and ours.

\section{Notation and basic definitions.}

2.1. Let $C_{k}$ denote complex Euclidean $k$-space. For points $z=\left(z_{1}, \cdots, z_{k}\right)$ and $w=\left(w_{1}, \cdots, w_{k}\right)$ of $C_{k}$ we denote their inner product $\sum_{1}^{k} z_{i} \bar{w}_{i}$ by $z \cdot w$, and write $r^{2}=|z|^{2}=z \cdot z$. By $\bar{z}$ we denote the $k$-tuple $\left(\bar{z}_{1}, \cdots, \bar{z}_{k}\right) . E_{k}$ denotes the set of entire functions in $C_{k}$, $f^{*}(z)$ always denotes the function $\mathrm{Cl}(f(\bar{z})$ ) ( $\mathrm{Cl}$ denotes complex conjugation), and $F_{k}$ denotes the set of $f \in E_{k}$ such that

$$
\|f\|^{2}=\pi^{-k} \int|f(z)|^{2} \exp \left(-|z|^{2}\right) d V
$$

is finite. Here the integration is over all of $C_{k}$, and $d V$ denotes the volume element (Lebesgue measure) in $C_{k}$, considered as a $2 k$-dimensional real Euclidean space:

$$
\begin{gathered}
d V=\prod_{m=1}^{k} d x_{m} d y_{m}=\prod_{m=1}^{k} r_{m} d r_{m} d \theta_{m}=\prod_{m=1}^{k} d A_{m} \\
z_{m}=x_{m}+i y_{m}=r_{m} \exp \left(i \theta_{m}\right) .
\end{gathered}
$$

It will sometimes be convenient to write

$$
d \sigma=\pi^{-k} \exp \left(-|z|^{2}\right) d V
$$

For two functions $f, g$ in $F_{k}$ we define their inner product

$$
\langle f, g\rangle=\int f(z) \mathrm{Cl}(g(z)) d \sigma .
$$

This inner product may be characterized also in the following way. Let $N$ (a "multi-index") denote the $k$-tuple $\left(n_{1}, \cdots, n_{k}\right)$ of nonnegative integers and write

$$
\begin{aligned}
& z^{N}=z_{1}^{n_{1}} \cdots z_{k}^{n_{k}}, \\
& N !=n_{1} ! \cdots n_{k} !, \quad|N|=\sum_{i=1}^{k} n_{i} .
\end{aligned}
$$


We partially order the $k$-tuples in the usual way: $M \leqq N$ if and only if $m_{i} \leqq n_{i}, i=1, \cdots, k$. Then, simple computations show

$$
\begin{gathered}
\left\langle z^{M}, z^{N}\right\rangle=0 \text { for } M \neq N, \\
\left\|z^{N}\right\|^{2}=N !
\end{gathered}
$$

In other words, the monomials $(N !)^{-1 / 2} z^{N}$ are orthonormal. This could also have been taken as our starting point; defining these monomials to be orthonormal one has then a positive definite inner product induced on the linear manifold of polynomials in $E_{k}$ and $F_{k}$ may be defined as the completion of the polynomials in the norm given by this inner product. From this it is clear that $F_{k}$ is a (complete) Hilbert space and may also be characterized as the set of $f=\sum a_{N} z^{N}$ in $E_{k}$ for which

$$
\|f\|^{2}=\sum N !\left|a_{N}\right|^{2}
$$

is finite.

From (10) one deduces that for each $w \in C_{k}$, the linear functional $f(w)$ is bounded on $F_{k}$. Hence $F_{k}$ possesses a reproducing kernel $K_{w}(z)=K(z, w)$ such that

$$
\left\langle f, K_{w}\right\rangle=\langle f(z), K(z, w)\rangle=f(w) .
$$

Using the orthonormal monomials as a basis one computes

$$
K_{w}(z)=\sum \frac{z^{N} \bar{w}^{N}}{N !}=\exp \left(\sum_{i=1}^{k} z_{i} \bar{w}_{i}\right)=e^{z \cdot w}
$$

(for further details concerning these basic relations see Bargmann [2]; for general background on reproducing kernels see [1]).

The relation (13) implies, by a well-known argument

$$
|f(z)|=O\left(\exp \left(r^{2} / 2\right)\right) \quad f \in F_{k} .
$$

From (14) we see that all functions in $F_{k}$ are of order $\leqq 2$. And from (1) we see that all functions of order $<2$ are in $F_{k}$, as well as some functions of order 2 .

We shall use the symbol $\|f\|$, for all $f \in E_{k}$, to denote $\left(\int|f(z)|^{2} d \sigma\right)^{1 / 2}$, whether the integral is finite or not. Finally, it will be convenient to consider that subclass $G$ of $F_{k}$ consisting of functions $\phi$ satisfying

$$
|\phi(z)|=O \quad\left(\exp \left(r^{2} / 2-A r\right)\right) \quad \text { for every } A>0 .
$$

This condition is equivalent to $\phi K_{w} \in F_{k}$ for all $w$.

2.2. Formal adjoints. The definitions in this paragraph are applicable to any Hilbert space with reproducing kernel. For economy 
of space we preserve however the notations of $F_{k}$. Let $A$ be a linear operator (in general, unbounded) whose domain of definition includes the set $L$ of finite linear combinations of kernel functions, and denote by $A_{1}$ the restriction of $A$ to $L$. Then $A_{1}^{*}$ is a closed extension of $A^{*}$, which we call the formal adjoint of $A$, and write $A^{\#}$. The function $g=A^{\#} f$ then satisfies

$$
g(w)=\left\langle f, A K_{w}\right\rangle
$$

In the case of $F_{k}$, if $\phi(z)$ satisfies $2.1(15)$, and $(\phi \cdot)$ denotes the operator "multiplication by $\phi$," the formal adjoint is denoted by $\phi^{*}(\partial / \partial z)$, and we have

$$
\phi^{*}\left(\frac{\partial}{\partial z}\right) f(z)=\int f(t) \operatorname{Cl}(\phi(t)) e^{z \cdot t} d \sigma
$$

When $\phi$ is a polynomial, $\phi^{*}(\partial / \partial z)$ coincides with the usual interpretation of this symbol.

2.3. Polynomial approximation. Let $m$ denote a positive Borel measure on $C_{k}$. We say that $m$ has the polynomial approximation (briefly, p.a.) property if and only if the polynomials are dense in the entire functions in the metric of $L^{2}(d m)$. In general, little is known about which measures have the p.a. property. Some results in the one-variable case may be found in the report of Mergelyan [11].

\section{Statement of results.}

TheOREM 1. For any $\phi$ satisfying 2.1(15), the following five statements are equivalent:

(i) The measure $|\phi|^{2} d \sigma$ has the p.a. property.

(ii) The orthogonal complement in $F_{k}$ of the null-space of $\phi^{*}(\partial / \partial z)$ is the set of $f \in F_{k}$ for which $f / \phi$ is entire.

(iii) $\phi^{*}(\partial / \partial z) f(z)=0$ and $f / \phi$ entire imply $f=0$.

(iv) The null-space of $\phi^{*}(\partial / \partial z$ ) is spanned (in the norm topology of $F_{k}$ ) by the exponential monomials (i.e. functions of the form $z^{N} e^{z \cdot a}$ ) it contains.

(v) $\phi^{*}(\partial / \partial z)$ is the adjoint of $(\phi \cdot)$.

THEOREM 2. Let $\phi$ be any exponential polynomial (i.e., finite linear combination of exponential monomials). Then $\phi$ has the properties enumerated in Theorem 1.

In this case $\phi^{*}(\partial / \partial z)$ is a differential-difference operator. Property (iv) is closely related to the theory of infinite order differential equations with constant coefficients. (References: Valiron [15], Muggli [12] for $k=1$; Ehrenpreis [3], Malgrange [9] for the many variable 
case. Our topology is however a different one.)

Thus far we know of no $\phi$ which satisfies 2.1(15) and does not have the properties (i)-(v). We have not, however, been able to extend Theorem 2 beyond exponential polynomials except for a few cases: (a) $\phi$ has no zeros, (b) some special cases for $k=1$, e.g. $\phi(z)=\sin z / z$.

The main tool in proving Theorem 2 is the following "isometry theorem," which is of independent interest. Identities bearing a formal similarity to (1) below have been used by Malgrange [11], Trèves [14, Lemma 1.3].

THEOREM 3. Let $\phi, f \in E_{k}$ and moreover for every $\epsilon>0,|\phi(z)|$ $=O\left(\exp \left(\epsilon r^{2}\right)\right)$. Then

$$
\|\phi f\|^{2}=\sum \frac{\left\|\phi^{(N) *}\left(\frac{\partial}{\partial z}\right) f(z)\right\|^{2}}{N !} .
$$

REMARK. Here $\phi^{(N) *}(\partial / \partial z)$ denotes the formal adjoint of $\left(\phi^{(N)} \cdot\right)$, and $\phi^{(N)}(z)$ denotes $\left(\partial / \partial z_{1}\right)^{n_{1}} \cdots\left(\partial / \partial z_{k}\right)^{n_{k}} \phi .(1)$ is understood to imply that if the left side is finite, $f$ belongs to the domain of each operator $\phi^{(N) *}$, and the series on the right converges.

Results are obtained also concerning the nonhomogeneous equation

$$
\phi^{*}\left(\frac{\partial}{\partial z}\right) f(z)=g(z)
$$

As is well known, the solution of (2) is intimately associated with the condition

(3) There is a constant $\delta>0$ such that $\|\phi f\| \geqq \delta\|f\|$.

This is equivalent to asserting that $(\phi \cdot)$ has a closed range.

THEOREM 4. Let $\phi(z)$ be an exponential polynomial with exponents $a_{i}$. Then $\phi$ satisfies (3) if and only if the convex hull of the points $a_{i}$ contains the origin of $C_{k}$.

If $\phi$ is an exponential polynomial satisfying the last condition we have then (in view of Theorem 2) : For every $g \in F_{k}$, (2) has a solution $f$, and indeed a unique solution $f_{0}$ which has the additional property that it is an entire multiple of $\phi$. This $f_{0}$ is also the solution of (2) of minimal norm, and is the orthogonal projection of $f$ on the range of $(\phi \cdot)$.

Theorem 3 enables us to compute the precise lower bound of the operator $(\phi \cdot)$ when $\phi$ is a polynomial. For example, when $\phi=P$ $=\sum_{1}^{k} z_{i}^{2}$ we show:

$$
\|P f\|^{2} \geqq 2 k\|f\|^{2}
$$


with equality only if $f$ is constant. Hence in $F_{k}$ the operator inverse to the Laplacian has the precise upper bound $(2 k)^{-1 / 2}$.

We have also,

THEOREM 5. Let $\phi(z)$ be any "pseudopolynomial," i.e.,

$$
\phi(z)=z_{1}^{n}+\sum_{i=1}^{n} f_{i}\left(z_{2}, \cdots z_{k}\right) z_{1}^{n-k}
$$

where $f_{i} \in F_{k-1}$. Then $\phi$ satisfies (3), with $\delta=1$. Hence, if also $\phi \in G,(2)$ has a solution for every $g \in F_{k}$.

4. Further questions. We see from Theorem 2 that, if $\phi$ is any exponential polynomial, the equation

$$
\phi^{*}\left(\frac{\partial}{\partial z}\right)(\phi f)=0
$$

together with $f \in F_{k}$, imply $f \equiv 0$. Since the operator $\phi^{*}(\partial / \partial z)$, interpreted as a differential-difference operator, is meaningfully defined for all entire functions, we may ask: does (1) have any nonnull entire solution $f$ ? That the answer is sometimes yes may be seen from the following example. Take $k=1, \phi(z)=e^{2 \pi i z}-1$. The question is then, is there $f \neq 0$ of period $2 \pi i$ which vanishes at the integers. Such an $f$ is easily constructed, namely

$$
f(z)=\left(1-e^{z}\right) \prod_{n=1}^{\infty}\left(1-e^{z-n}\right)\left(1-e^{-z-n}\right) .
$$

It is easily seen that this product converges uniformly on compact sets, and so defines an entire function. Moreover $|f(z)|$ $=O\left(\exp \left((1 / 2+\epsilon) r^{2}\right)\right)$ for every $\epsilon>0$, i.e., $f$ "just misses" being in $F_{k}$.

On the other hand, there are certain $\phi$ for which (1) has no nonnull entire solution. We can show this is the case when (i) $\phi$ is a homogeneous polynomial, (ii) $\phi$ is a polynomial with at most $k$ nonvanishing coefficients (e.g., for $k=3$ such a polynomial as $z_{1}^{2}+z_{2} z_{3}-z_{3}^{3}$ ). Possibly this is true for all polynomials (in one variable this is trivially true).

We mention one final question: the theorems of this paper establish relations between the operator $\phi^{*}(\partial / \partial z)$ and the set in $C_{k}$ where $\phi$ vanishes. This suggests the possibility of replacing the set where $\phi$ vanishes by the set where $\psi$ vanishes, where $\psi$ is some other entire function. Of course then the special role played by the space $F_{k}$ disappears. A typical problem could be: given $\phi$, for which entire func- 
tions $\psi$ do we have $\phi^{*}(\partial / \partial z)(\psi f)=0, f$ entire, implies $f \equiv 0$ ? Already the case $k=2, \phi=z_{1}^{2}+z_{2}^{2}$ presents formidable difficulties (this is essentially the problem of characterizing the level curves of a harmonic function; some results have been obtained in [6]).

\section{BIBLIOGRAPHY}

1. N. Aronszajn, La théorie des noyaux réproduisants et ses applications. I, Proc. Cambridge Philos. Soc. 39 (1943), 133-153.

2. V. Bargmann, On a Hilbert space of analytic functions and an associated integral transform, Comm. Pure Appl. Math. 14 (1961), 187-214.

3. L. Ehrenpreis, Mean periodic functions. I, Amer. J. Math. 77 (1955), 293-328.

4. E. Fischer, Über algebraische Modulsysteme und lineare homogene partielle Differentialgleichungen mit konstanten Koeffizienten, J. für Math. 140 (1911), 48-81.

5. — - Über die Differentiationsprozesse der Algebra, J. für Math. 148 (1917), $1-78$.

6. L. Flatto, D. J. Newman and H. S. Shapiro, The level curves of harmonic functions, Trans. Amer. Math. Soc. 123 (1966). 1931.

7. E. W. Hobson, The theory of spherical and ellipsoidal harmonics, Cambridge,

8. J. Horvath, Basic sets of polynomial solutions for partial differential equations, Proc. Amer. Math. Soc. 9 (1958), 569-575.

9. B. Malgrange, Existence et approximation des solutilns des équations aux dérivées partielles et des équations de convolution, Ann. Inst. Fourier 6 (1955-1956), 271-355.

10. - - Sur une inégalité de F. Trèves, Math. Z. 72 (1959), 184-188.

11. S. N. Mergelyan, On the completeness of systems of analytic functions, Uspehi Mat. Nauk. 8 (1953), 3-63 (Russian).

12. H. Muggli, Differentialgleichungen unendlich hoher Ordnung, Comment. Math. Helv. 14 (1942), 381-393.

13. D. J. Newman and H. S. Shapiro, A Hilbert space of entire functions related to the operational calculus, University of Michigan, Ann Arbor, 1964, 92 pp. (mimeographed notes).

14. F. Trèves, Lectures on linear partial differential equations with constant coefficients, Rio de Janeiro, 1961, (mimeographed lecture notes).

15. G. Valiron, Sur les solutions des équations differentielles linéaires d'ordre infini et a coefficients constants, Ann. Sci Ecole Norm. Sup. (3) 46 (1929), 25-53.

Yeshiva UNIVERSITY, AND

UNIVERSITY OF MICHIGAN 